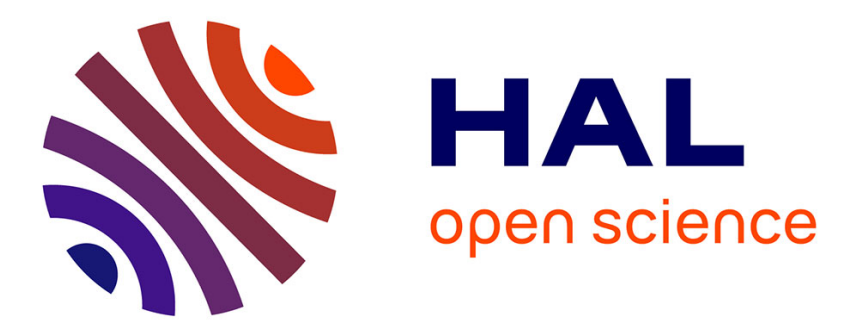

\title{
Refractory vertically aligned carbon nanotube-boron nitride nanocomposites for scalable electrical anisotropic interconnects
}

Qi Ming Zou, Lei Min Deng, Pei Xun Fan, da Wei Li, Chen Fei Zhang, Li Sha Fan, Lan Jiang, Jean-François Silvain, Yong Feng Lu

\section{To cite this version:}

Qi Ming Zou, Lei Min Deng, Pei Xun Fan, da Wei Li, Chen Fei Zhang, et al.. Refractory vertically aligned carbon nanotube-boron nitride nanocomposites for scalable electrical anisotropic interconnects. ACS Applied Nano Materials, 2019, 2 (1), pp.100-108. 10.1021/acsanm.8b01727 . hal-02104869

\section{HAL Id: hal-02104869 \\ https://hal.science/hal-02104869}

Submitted on 1 Oct 2020

HAL is a multi-disciplinary open access archive for the deposit and dissemination of scientific research documents, whether they are published or not. The documents may come from teaching and research institutions in France or abroad, or from public or private research centers.
L'archive ouverte pluridisciplinaire HAL, est destinée au dépôt et à la diffusion de documents scientifiques de niveau recherche, publiés ou non, émanant des établissements d'enseignement et de recherche français ou étrangers, des laboratoires publics ou privés. 


\section{Refractory Vertically Aligned Carbon Nanotubes-Boron \\ 2 Nitride Nanocomposites for Scalable Electrical 3 Anisotropic Interconnects}

4 Qi Ming Zou, ${ }^{\dagger, \pm}$ Lei Min Deng, $,{ }^{\dagger},{ }^{\prime \prime}$ Pei Xun Fan, ${ }^{\dagger} * *$ Da Wei Li, ${ }^{\dagger}$ Chen Fei Zhang, ${ }^{\dagger}$ Li Sha Fan, ${ }^{\dagger}$

5 Lan Jiang, ${ }^{£}$ Jean-Francois Silvain, ${ }^{\S}$ and Yong Feng $L u^{\dagger, *}$

$6{ }^{\dagger}$ Department of Electrical and Computer Engineering, University of Nebraska-Lincoln,

$7 \quad$ Lincoln, NE 68588-0511, United States

$8 \quad{ }^{£}$ School of Mechanical Engineering, Beijing Institute of Technology, Beijing, 100081, China

9 §Institut de Chimie de la Matière Condensée de Bordeaux, Avenue du Docteur Albert Schweitzer

10 F-33608 Pessac Cedex, France

11 "Wuhan National Laboratory for Optoelectronics, Huazhong University of Science and 12 Technology, Wuhan, 430074, China

KEYWORDS: anisotropic, ceramic nanocomposites, vertically aligned carbon nanotube, electrical interconnects, boron nitride

ABSTRACT: Traditional metal interconnect technology faces several challenges when scaling

17 down, such as electromigration and poisoning. Carbon nanotubes (CNTs) have been introduced in an attempt to solve these problems while providing on par performance. However, unexpected issues, such as great difficulty in manufacturing perfectly aligned CNTs and the undesired current 
1 leakage caused by electron percolation, still exist. In this work, we present novel vertically aligned

2 CNT (VACNT)-based nanocomposites utilizing hexagonal boron nitride (h-BN) as intertube

3 insulating/shielding layers that can be prepared in a scalable and controllable fashion. This

4 composite material inherits the full advantages of the directional conductivity of VACNTs which

5 are strongly enhanced by the intertube h-BN layer and demonstrate excellent electrical anisotropy.

6 This composite material achieves conductivities of 1060.43 and $4.43 \mathrm{~S} \cdot \mathrm{m}^{-1}$ along the directions

7 parallel with and perpendicular to the VACNTs, respectively, while the previously reported

8 electrical conductivity of CNT-polymer and CNT-ceramic counterparts are well below $10^{-3} \mathrm{~S} \cdot \mathrm{m}^{-1}$

9 isotropically. Due to its refractory ability, the h-BN layer can also protect the prepared

10 nanocomposites from harsh oxidation and erosion, showing ultrahigh stability up to $1400{ }^{\circ} \mathrm{C}$.

11 These results reflect a giant step toward a simple, turnkey solution to an advanced CNT-based

12 composite material for future electrical interconnect applications.

\section{INTRODUCTION}

14 Modern interconnect technology was introduced into the fabrication of printed circuit boards 15 (PCBs) and very-large-scale integration (VLSI) at least two decades ago. ${ }^{1-5}$ It allowed PCBs to be

16 designed with much higher routing density ${ }^{6}$ and revolutionized the VLSI industry by significantly 17 increasing the performance of chips. ${ }^{7}$ As feature sizes continuously shrink, interconnect 18 technology demands much denser routing to decrease costs and avoid reliability risks. ${ }^{6}$ While 19 copper $(\mathrm{Cu})$ interconnects are dominating the market, they require barrier layers or special alloys 20 to prevent poisoning when ramping up the routing density. ${ }^{1}$ In addition, $\mathrm{Cu}$ interconnects suffer 
1 electromigration (EM) which worsens when the current density increases. ${ }^{8}$

2 Carbon nanotubes (CNTs) have recently been recognized as a promising alternative to $\mathrm{Cu}$

3 interconnects. ${ }^{9,10}$ It has been suggested that CNTs are the interconnect material of the future ${ }^{11}$ based

4 on their outstanding electrical properties as well as extraordinary failure current densities $\left(>10^{9}\right.$

$\left.5 \mathrm{~A} \cdot \mathrm{cm}^{-2}\right) \cdot{ }^{9,10,12-15}$ In addition, CNTs have the ability to carry huge current density without heat sinks

6 because of their exceptional thermal conductivity. ${ }^{16,17}$ However, great difficulty exists in

7 manufacturing perfectly aligned CNTs, which are beneficial for minimizing the conducting

8 resistance. ${ }^{18,19}$ When stacking clustered CNTs, the intertube conduction becomes significant due

9 to electron percolation, causing current leakage toward unwanted directions. ${ }^{20}$ Consequently,

10 CNT-dielectric composite systems have been introduced in order to manipulate the percolation by

11 fine controlling the electrical percolation threshold (EPT) to prevent intertube conducting behavior,

12 at the expense of high electrical conductivity, however, when compared to bare CNT networks. In

13 such systems, consisting of a mix of dielectric and conductive materials, the formation of

14 conductive pathways in the form of clusters of aligned CNTs inside the matrix is governed by

15 classical percolation theories. ${ }^{21,22}$ The conductivity of CNT-dielectric composite systems depends upon the alignment of nanotubes, with the highest conductivity being obtained via highly aligned

17 CNTs. ${ }^{18}$ On this basis, a promising solution for addressing all of the stated issues is to combine vertically aligned carbon nanotubes (VACNTs) with appropriate dielectric materials.

As a thermally and chemically resistant refractory compound of boron (B) and nitrogen (N), boron nitride $(\mathrm{BN})$ came to our attention. It exists in various crystalline forms that are isoelectronic 21 to a similarly structured carbon lattice. ${ }^{23}$ Among those forms, hexagonal boron nitride (h-BN) 
1 demonstrates excellent anticorrosion ability, ${ }^{24}$ thermal stability, ${ }^{25}$ and, as a wide-bandgap

2 semiconductor material, a decent dielectric property. ${ }^{26}$ However, the fabrication of VACNT-BN

3 remains a huge challenge. As we know, the average intertube distance in VACNT arrays is less

4 than $10 \mathrm{~nm}$, which greatly limits the infiltration of the majority of materials into the VACNT

5 array. $^{27,28}$ Although thermal chemical vapor deposition (CVD), ${ }^{28-30}$ and atomic layer deposition

$6(\mathrm{ALD})^{31}$ have been reported to be effective in regard to the infiltration of materials into dense

7 VACNT arrays, successful preparation of VACNT-BN nanocomposites with electrical anisotropy

8 has rarely been realized.

9 In this work, we developed an efficient approach to the controllable fabrication of VACNT-BN

10 nanocomposites, which utilized VACNTs as a conductive network and h-BN as an intertube

11 shielding layer, via a single-step CVD process. Vertically aligned carbon nanotubes, rather than

12 other carbon (C) nanomaterials, were chosen as the conducting framework due to their highly

13 aligned structure which guarantees the conductive pathway after the infiltration of h-BN into the

14 matrices and minimizes the thermal stress at elevated temperatures. ${ }^{17}$ The as-prepared VACNT-

15 BN nanocomposites exhibited highly anisotropic conductivity in the network, the mechanism

16 behind which was studied and attributed to the different electrical percolation thresholds in

17 different directions as a nature of the VACNT-BN nanocomposites. The nanocomposites also have

18 excellent high-temperature antioxidative ability (up to $1400^{\circ} \mathrm{C}$ ) and erosion resistance, suggesting

19 that the prepared VACNT-BN nanocomposites have not only great potential for next-generation

20 electrical interconnects but much more potential is yet to be explored.

21 


\section{METHODS}

2 Fabrication of ultralong VACNTs via CVD: Ultralong VACNTs were fabricated in a similar

3 fashion as reported previously. ${ }^{28}$ The growth of VACNTs was carried out in a two-zone CVD

4 furnace (MTI Corporation, OTF-1200X) after appropriate catalysts were sputtered onto the

5 substrate. Two different types of VACNT samples were prepared: VACNT films and cubic-

6 patterned VACNTs.

7 Fabrication of VACNT-BN nanocomposites: VACNT-BN nanocomposites were prepared via

8 CVD. The VACNT samples were placed inside a tube furnace with customized gas inlets and were

9 heated in an ammonia flow until $1100{ }^{\circ} \mathrm{C}$ was reached. Then, boron trifluoride was introduced into

10 the chamber over a time period as long as $180 \mathrm{~min}$. During the $\mathrm{BN}$ deposition, the chamber

11 pressure was maintained at $\sim 2.3$ Torr.

12 Fabrication of VACNT-BN electrical conductivity test benches and I-V measurements:

13 VACNT-BN samples were thinned and lifted out of the bulk matrices via FIB (FEI, Helios

14 NanoLab $^{\mathrm{TM}}$ 660). Electrode patterns were prepared via photolithography (SUSS MicroTec Group,

15 MJB4 Mask Aligner) and Au was then sputtered by a magnetron sputtering system (AJA

16 International, Inc., ATC-Orion $5 \mathrm{UHV}$ ) to obtain Au electrodes. The VACNT-BN lifted out were

17 then transferred onto the Au electrodes. The I-V characteristics were measured by a probe station

18 (Cascade MPS 150) and a semiconductor parameter analyzer (Agilent 4155C) at room temperature. 
1 High-temperature antioxidative ability and thermal stability test: For antioxidative ability

2 testing, the as-prepared h-BN film and VACNT-BN nanocomposites were oxidized in a muffle

3 furnace (Thermolyne ${ }^{\mathrm{TM}}$ Benchtop $1100{ }^{\circ} \mathrm{C}$ ) at different temperatures until $1100{ }^{\circ} \mathrm{C}$. Further

4 oxidation from $1100{ }^{\circ} \mathrm{C}$ to $1400{ }^{\circ} \mathrm{C}$ was carried out in a tube furnace (MTI Corporation, GSL-

5 1500X-RTP50), with an oxygen partial pressure of 100 mTorr. High-temperature gas erosion

6 resistance of VACNT-BN was carried out by an oxy-acetylene torch, for as long as $126 \mathrm{~min}$

7 (Figure S16, supporting material).

8 Characterization: Raman spectral analysis of the samples was performed in a micro-Raman

9 system (Renishaw, inVia ${ }^{\mathrm{TM}}$ Plus). A $514 \mathrm{~nm} \mathrm{Ar}+$ laser with a power of $\sim 3 \mathrm{~mW}$ was used to excite

10 the Raman scattering. Raman spectra and mapping were then collected through a 50× objective

11 lens with an accumulated time of $10 \mathrm{~s}$ at each position. Scanning electron microscopy (FEI, Helios

12 NanoLab 660) was used to study the morphologies of the as-prepared VACNT-BN

13 nanocomposites. Energy-dispersive x-ray was carried out via this SEM system as well.

14 Transmission electron microscopy ( FEI, Tecnai Osiris S/TEM) was used to study the

15 nanostructures of the grown BN and VACNT-BN samples. Energy-dispersive x-ray, EELS, and

16 HAADF analysis were also carried out via this scanning TEM system. 


\section{3. RESULTS AND DISCUSSION}

\section{$2 \quad$ 3.1. Design and Fabrication}

\section{3.1.1. Growth of thick boron nitride layers}

4 In this study, thick boron nitride films were prepared via CVD. Hexagonal boron nitride film as

$5 \quad$ thick as $11.8 \mu \mathrm{m}$ (Figure S1, supporting material) was successfully obtained in a fashion similar to

6 the one reported previously, ${ }^{32}$ with a growth time of $180 \mathrm{~min}$, corresponding to a fast growth rate

7 of $\sim 4 \mu \mathrm{m} / \mathrm{h}$. Interestingly, two different kinds of h-BN structures can be distinguished from the

8 cross-sectional scanning electron microscopy (SEM) image shown in Figure S1b. To chemically

9 examine the h-BN film grown, cross-sectional Raman mapping was obtained and is shown in

10 Figure S2 to provide more structural and quality information on the as-grown, thick h-BN film.

11 The Raman mapping of peak intensity, peak position, and peak width of the as-grown h-BN were

12 compared (Figure S2c-S2h, respectively). The peak intensity was strong in the top section (layered)

13 of the h-BN and was slightly lower in the bottom section (particle-like) of h-BN. However, there

14 was not much difference in the peak position and width of the $E_{2 g}$ band across the cross-section,

15 suggesting an overall uniform distribution of quality. ${ }^{33}$ In order to further confirm the crystal

16 structure of these two different layers, a cross-sectional transmission electron microscopy (TEM)

17 sample $\sim 25 \mathrm{~nm}$ thick was prepared via focused ion beam (FIB), shown in Figure S3. As can be

18 seen, the top BN layer shows a highly oriented, layered structure, as typical h-BN. On the contrary,

19 the bottom BN layer contained many different polycrystalline orientations, which was confirmed

20 to be amorphous BN. The electron energy loss spectroscopy (EELS) spectrum, shown in Figure 
1 S3e, further confirms the B-N structure.

\section{3.1.2. Controlled growth of boron nitride on vertically aligned carbon nanotubes}

3 We applied the same h-BN growth technique directly onto VACNTs instead of silicon

4 dioxide/silicon $\left(\mathrm{SiO}_{2} / \mathrm{Si}\right)$. As previously reported, the infiltration of materials into dense VACNT

5 arrays is extremely challenging; and the thermal CVD system was fine-tuned to ensure a

6 homogeneous dispersion of h-BN inside the VACNT arrays. ${ }^{28}$ Figure 1a illustrates the controlled

7 fabrication process of VACNT-BN nanocomposites. In order to fabricate VACNT-BN samples,

8 both VACNT films of different thicknesses (Figure 1a) and cuboid-patterned VACNTs (Figure 1b

9 and 1c) were prepared for the h-BN infiltration. Optimized h-BN growth parameters were used for

10 the infiltration; and the corresponding x-ray diffraction (XRD) results for VACNTs, h-BN film,

11 and VACNT-BN (shown in Figure S4) were used to confirm the composite structure. As can be

12 seen, both h-BN film and VACNT-BN exhibited a main feature of (002) while only VACNT-BN

13 exhibits (004). It is reported that the (002) plane of h-BN exhibits similar properties of a graphene-

14 like plane. ${ }^{34}$ While the (004) plane of h-BN is parallel to the (002) plane, the XRD intensity of

15 these two planes can be largely different due to different facets that are exposed in the crystals. ${ }^{35}$

16 This indicates that the crystal alignment of $\mathrm{h}-\mathrm{BN}$ film and that of VACNT-BN are different, which

17 could be caused by a much more complicated surface condition of VACNTs for BN to grow.

The well-controlled growth of VACNT-BN yielded short (Figure S5) and ultralong (Figure S6

19 and S7) VACNT-BN nanocomposites. Figure S5 is a cross-sectional SEM image of an as-grown,

20 short VACNT-BN sample. An excessive layer of h-BN can be seen above the lower VACNT-BN 
1 section. The VACNTs were heavily covered by h-BN as the fiber structures visually became much

2 thicker. Figures S6 and S7 demonstrate the uniformity of the ultralong VACNT-BN

3 nanocomposites along all directions. As an example, a 1.3-mm-long VACNT-BN cuboid was cut

4 in half; and the cross-sectional SEM images for vertical (Figure S6) and horizontal (Figure S7)

5 directions of the sample are presented. As shown in these results, the uniformity in macroscale

6 turns out to be excellent.

7

(a)

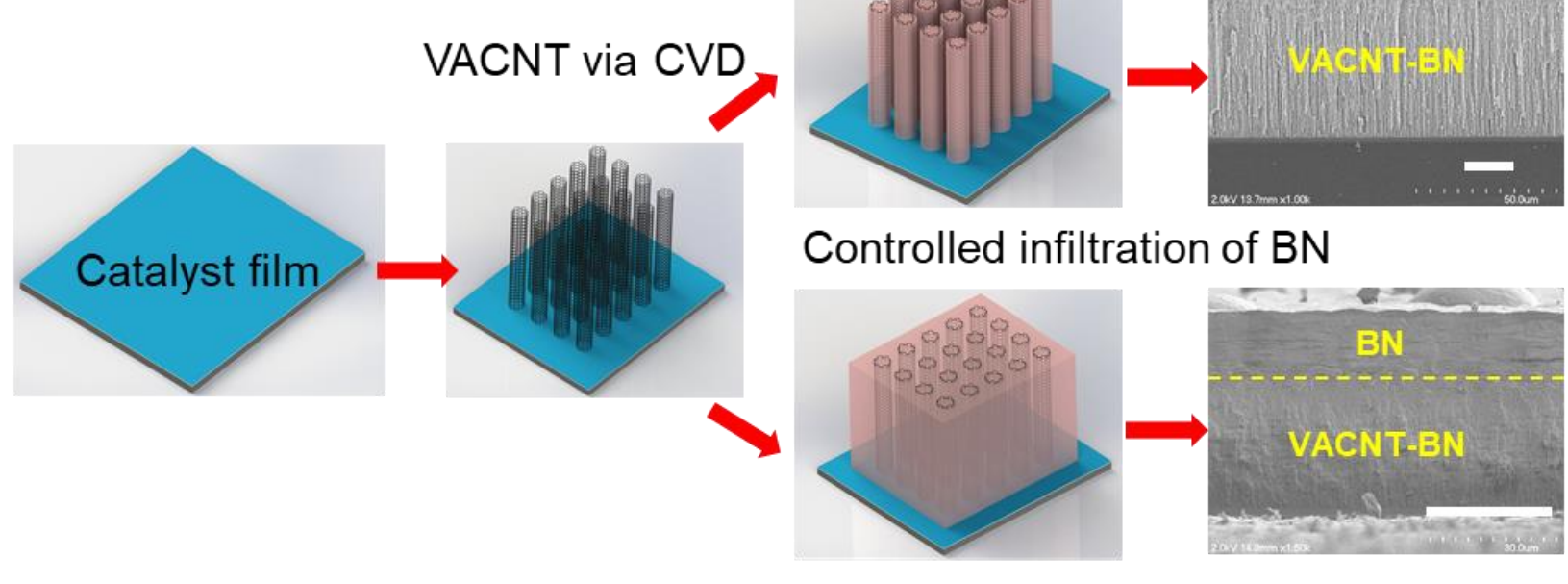

(b)

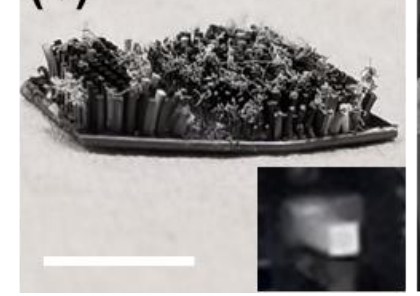

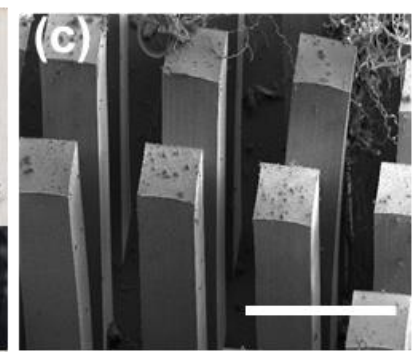

(d)

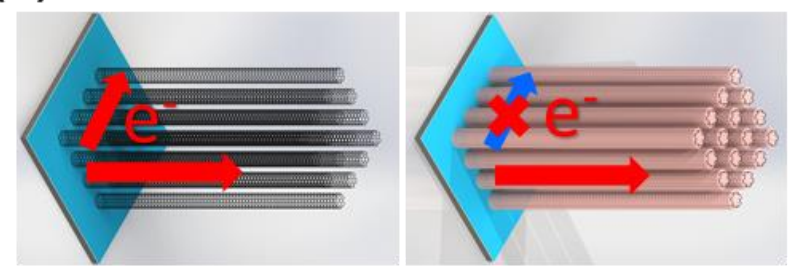

9 Figure 1. Illustration of controlled fabrication process and electron percolation mechanism. (a) The controlled fabrication process of VACNT-BN. Two cross-sectional SEM images demonstrate two different types of VACNT-BN film samples. (b) Optical image and (c) SEM image of cuboid-patterned VACNT-BN sample. Inset in (b) shows one single magnified VACNT-BN cuboid. (d) Different electron percolation conditions in VACNT and VACNT-BN. Scale bars for (a) $20 \mu \mathrm{m}$, (b) $1 \mathrm{~cm}$, and (c) $50 \mu \mathrm{m}$. 
2 In our VACNT-BN system, the composite mixture was highly oriented by utilizing the VACNT as

3 a template. Without insulating layers outside the VACNTs, current flowed along the direction of

4 the VACNTs and intertube at the same time due to electron percolation (illustration Figure 1d). In

5 order to achieve highly anisotropic conductivity, we engineered the VACNT-BN nanocomposites,

6 based on the dielectric nature of h-BN. Dense VACNT arrays were used as the conducting network

7 and building template, into which we infiltrated $\mathrm{BN}$ in a controlled manner. Hexagonal boron

8 nitride served as an intertube shielding layer, increasing the electron percolation threshold of the

9 VACNTs while maintaining the pristine conducting ability along the direction of the VACNTs.

\subsection{Structural and Chemical Characterization}

11 Although the graphene-like structure of the wall of VACNTs can act as a template for the

12 nucleation of h-BN, achieving a stable and functional combination of these two materials remains

13 a great challenge. ${ }^{29,36-39}$ To explore this matter, we first looked into the structure, in macroscale,

14 of the VACNT-BN nanocomposites. Cross-sectional SEM images of them are presented in Figure

15 2. During the growth of h-BN, gas feedstocks first infiltrated into the VACNT array, forming h-

16 BN layers which eventually accumulated on the top surface, resulting in the formation of a thick

$17 \mathrm{~h}-\mathrm{BN}$ layer on top. As can be seen in Figure $2 \mathrm{a}$ and $2 \mathrm{~b}$, the h-BN layer on top exhibited a layered

18 structure while the VACNT-BN region maintained its original vertically aligned feature (Figure

19 2d). The magnified SEM image (Figure 2b) gives a clearer view of the layered h-BN structure.

20 Different boundaries are outlined by the yellow dashed lines, indicating the evolution in size of $h$ - 
1 BN layers, from small fractions to bigger plains. The small fractions are considered to be the consequence of direct growth on the top surface of the VACNT array, which was very rough. In addition, a clear boundary of VACNT-BN and h-BN regions can be identified (Figure 2c).
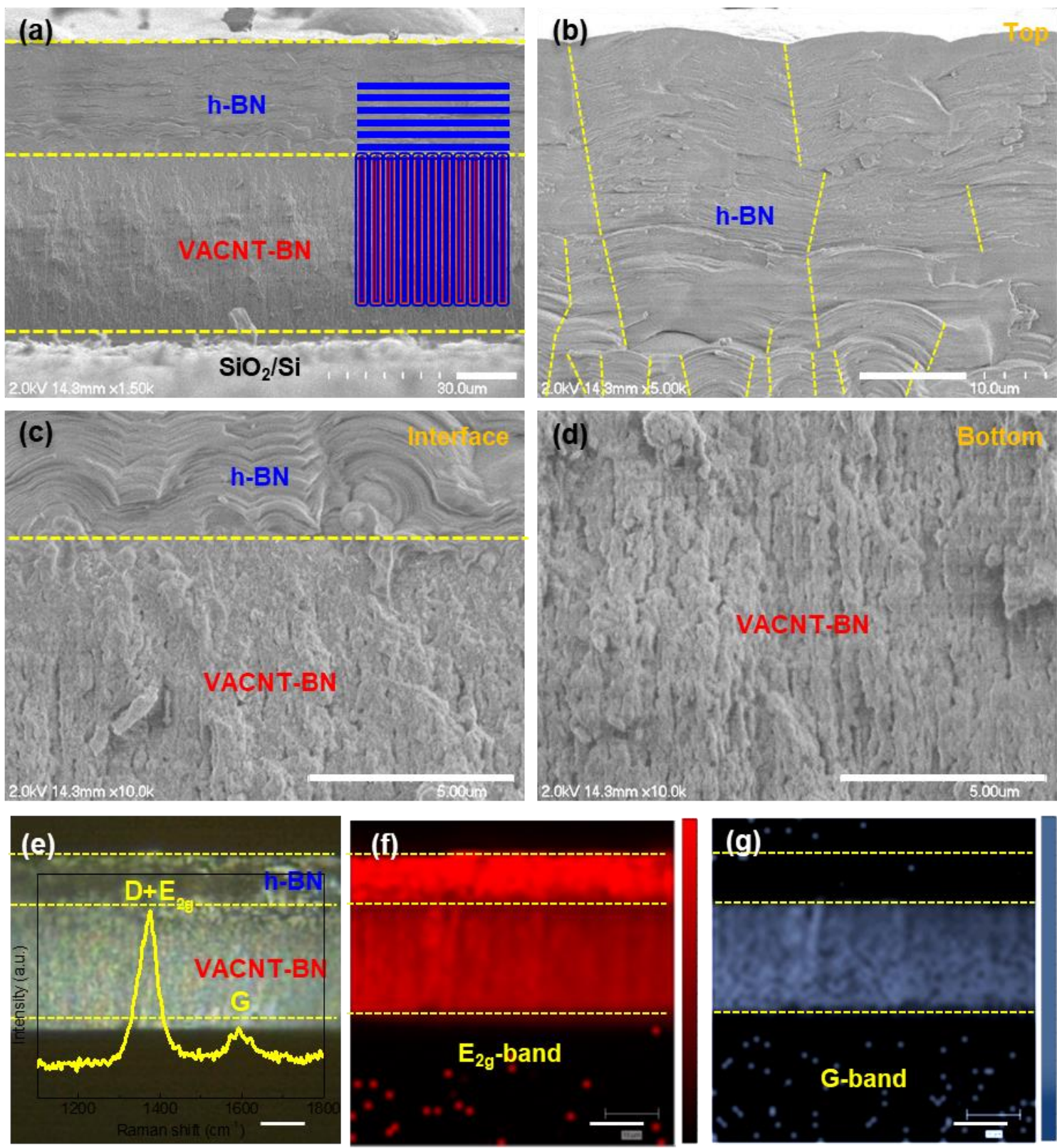

Figure 2. Microscale structural and chemical analysis of prepared VACNT-BN nanocomposites. (a) Low magnification cross-sectional SEM images of VACNT-BN. Scale bar: $10 \mu \mathrm{m}$. (b-d) High magnification crosssectional SEM images corresponding to the top, interfacial, and bottom area of (a), respectively. Scale bar: $5 \mu \mathrm{m}$. (e) Optical microscopic image of the sample. (f,g) Cross-sectional Raman intensity mapping of (f) $9 \quad$ E2g band and $(\mathrm{g}) \mathrm{G}$ band of the area shown in (e). Scale bars for $(\mathrm{e}-\mathrm{g}): 10 \mu \mathrm{m}$. 
To help better evaluate these different structures, Raman mapping of this cross-section area

was carried out, as shown in Figure 2e-2g. A typical Raman spectrum of VACNT-BN is shown in the inset of Figure 2e. For the VACNT-BN region, the $\mathrm{E}_{2 \mathrm{~g}}$ band was separated from the combined $\mathrm{D}+\mathrm{E}_{2 \mathrm{~g}}$ band via Lorentzian fitting. As can be seen from the $\mathrm{E}_{2 \mathrm{~g}}$ band intensity mapping, which represents the h-BN distribution along the cross-section, the top h-BN layer demonstrated a stronger signal than the VACNT-BN section. This was the result of peak shifting caused by the mixing of two materials. ${ }^{40}$ As for the G-band intensity mapping, which represents the VACNTs, it proved the existence of VACNTs under the heavy h-BN coating on the surface. A similar analysis was carried out on shorter VACNTs as well, and the results were consistent (Figure S8).

Although the above results visually and chemically confirmed that the h-BN was infiltrated inside the VACNT matrices, the combination between every single VACNT and the h-BN shielding layer was yet to be determined. Therefore, we looked further into this matter via various characterization techniques of scanning TEM (STEM). First, from a chemistry point of view, as can be seen in Figure 3b-3d, the results of energy dispersive spectroscopy (EDS) for (b) boron, (c) carbon, and (d) nitrogen mapping are presented. The high-angle annular dark field (HAADF) image of the sampled area is shown in Figure 3a as a reference. Strong signals for all three elements were obtained with good contrast. The distribution for both $\mathrm{B}$ and $\mathrm{N}$ was consistent with $\mathrm{C}$, with no overlapping in the intertube space, indicating that the h-BN was only on the sidewall of the VACNTs. Taking full advantage of the better contrast provided by the HAADF image ${ }^{41,42}$ of the sample (Figure 3a), the h-BN coating on VACNT fibers was confirmed. To narrow down the scope and further look into one single VACNT, a HAADF (Figure 3e) image of one single VACNT is 

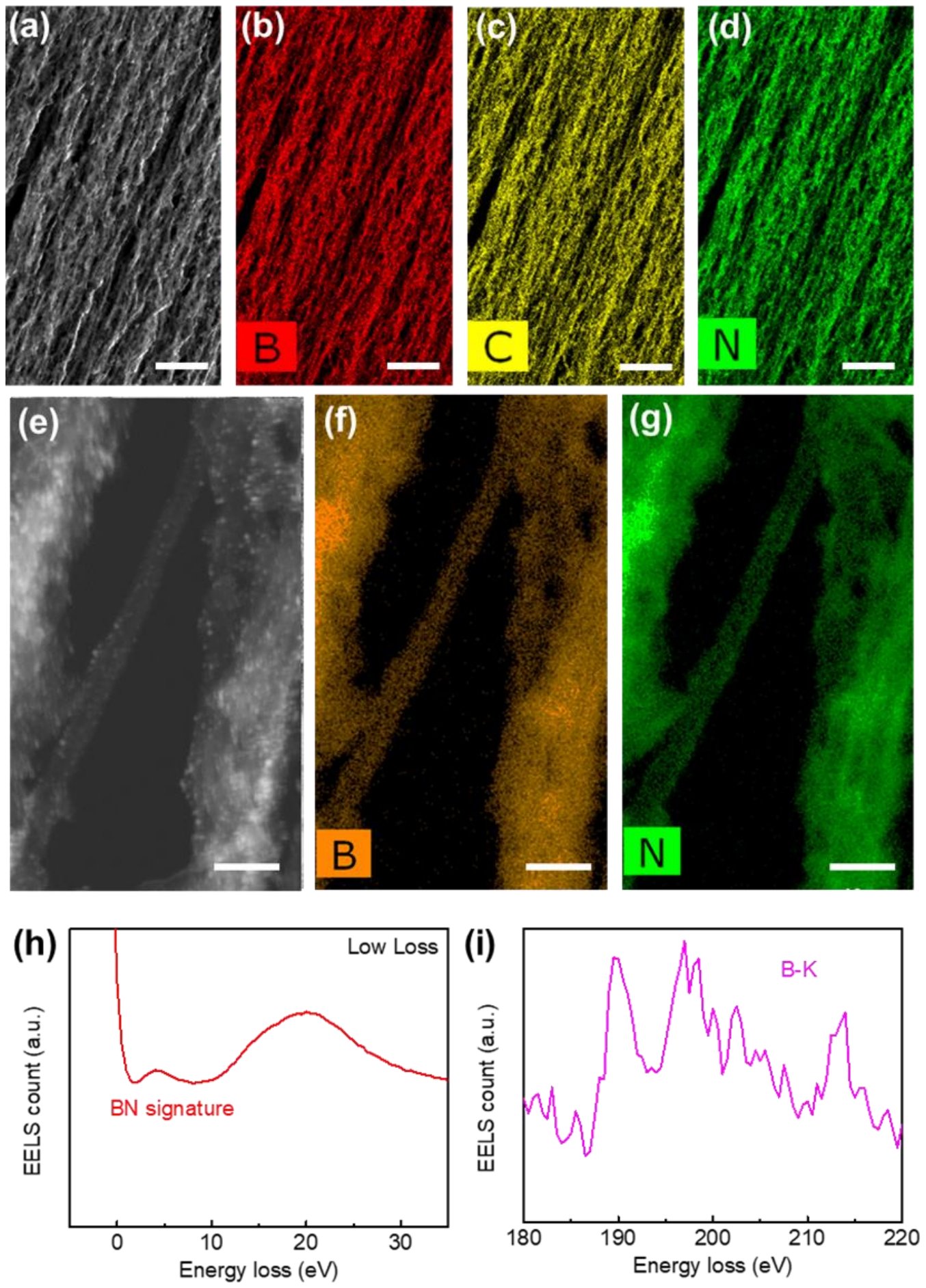

Figure 3. Nanoscale elemental analysis of prepared VACNT-BN nanocomposites. (a) HAADF image, 3 (b) boron, (c) carbon, and (d) nitrogen EDS mapping of VACNT-BN fibers. Scale bars: (a-d) $1 \mu \mathrm{m}$. 4 (e) HAADF image, (f) boron, and (g) nitrogen EDS mapping of a single VACNT-BN. Scale bars: (e-g) 40 $5 \mathrm{~nm}$. (h) EELS low loss spectrum of VACNT-BN. (i) BN signature band in EELS spectrum of the VACNT-BN 6 sample. 
shown, alongside the EDS mapping of B (Figure 3f) and N (Figure 3g).as well. As can be seen, a single VACNT was suspended between surrounding structures, and both B and N elements were uniformly covering the VACNT wall.

However, some previous reports suggested that at elevated temperatures, $\mathrm{C}, \mathrm{B}$, and $\mathrm{N}$ can form complex chemical species that are not desired in our study. ${ }^{43-46}$ To investigate this matter, EELS of the VACNT-BN sample of a larger area was collected. First, the low loss map of the nanocomposites is shown in Figure 3h, which suggests results consistent with the reported h-BN

8 signature. ${ }^{47,48}$ Another set of unique B-K signature peaks that only exist in BN was also identified, as shown in Figure 3i. The full EELS spectrum of the sample and additional features associated with $\mathrm{C}$ and $\mathrm{N}$ are shown in Figure S9. Interestingly, neither BN nor B-C-N features were identified in the spectrum. These results confidently indicate that the VACNT-BN nanocomposites we on the wall of the VACNT-BN (Figure 4b) and the wall of the VACNT-BN is slightly distorted. These features exhibited a similar graphene-like layered structure. To better evidence the existence of the h-BN, we took higher magnification HRTEM images of both VACNT-BN and h-BN film to compare with each other, and the figures have been added in the supporting material (figure S10) 
1 and is also shown below. High magnification HRTEM of the outside layers that are suspected to

2 be h-BN is shown in figure S10a. As can be seen, in addition to the already identified layered

3 structure, the atom-atom distance is roughly measured to be $2.6 \AA$ and the distance consisting of

4 two layers is around 5.9 $\AA$. To verify these results, we compared them to the HRTEM of h-BN

5 shown in figure S10b. It demonstrates a similar graphene-like layered structure, with a layer

6 thickness of roughly $3 \sim 4 \AA$, which is consistent to both shown in figure $4 \mathrm{~b}$ and reported

7 results..$^{30,33,49}$ Combining this structural consistency and the elemental characterization provided

8 by EELS and EDS, we are therefore positive that these graphene-liked layers outside the VACNT

9 wall are h-BN..$^{36,39,50,51}$ In addition, large h-BN nucleation sites were noted (dark round dots). The

10 existence of these large spots is believed to correspond to the white features seen in the HAADF

11 image (Figure 3e). Based on the location of these features, the graphene-like layers were

12 determined to first form by the wall of a VACNT, which served as a template. These layers then

13 served as transition layers for the larger scale nucleation of h-BN dots. This growth mechanism

14 allowed a chemically and structurally stable combination of VACNTs and BN. ${ }^{36,48}$ However, as

15 shown in Figure 4b, the wall of VACNT after BN growth has been slightly distorted due to the

16 formation of these BN layers. Comparing to other reports with similar CNT-BN structures, ${ }^{30,52}$ our

17 results show discontinued BN layers covering the VACNTs instead of a few smooth epitaxial layers.

18 This discontinued formation of BN may have been caused by the purposed nucleation mechanism. 

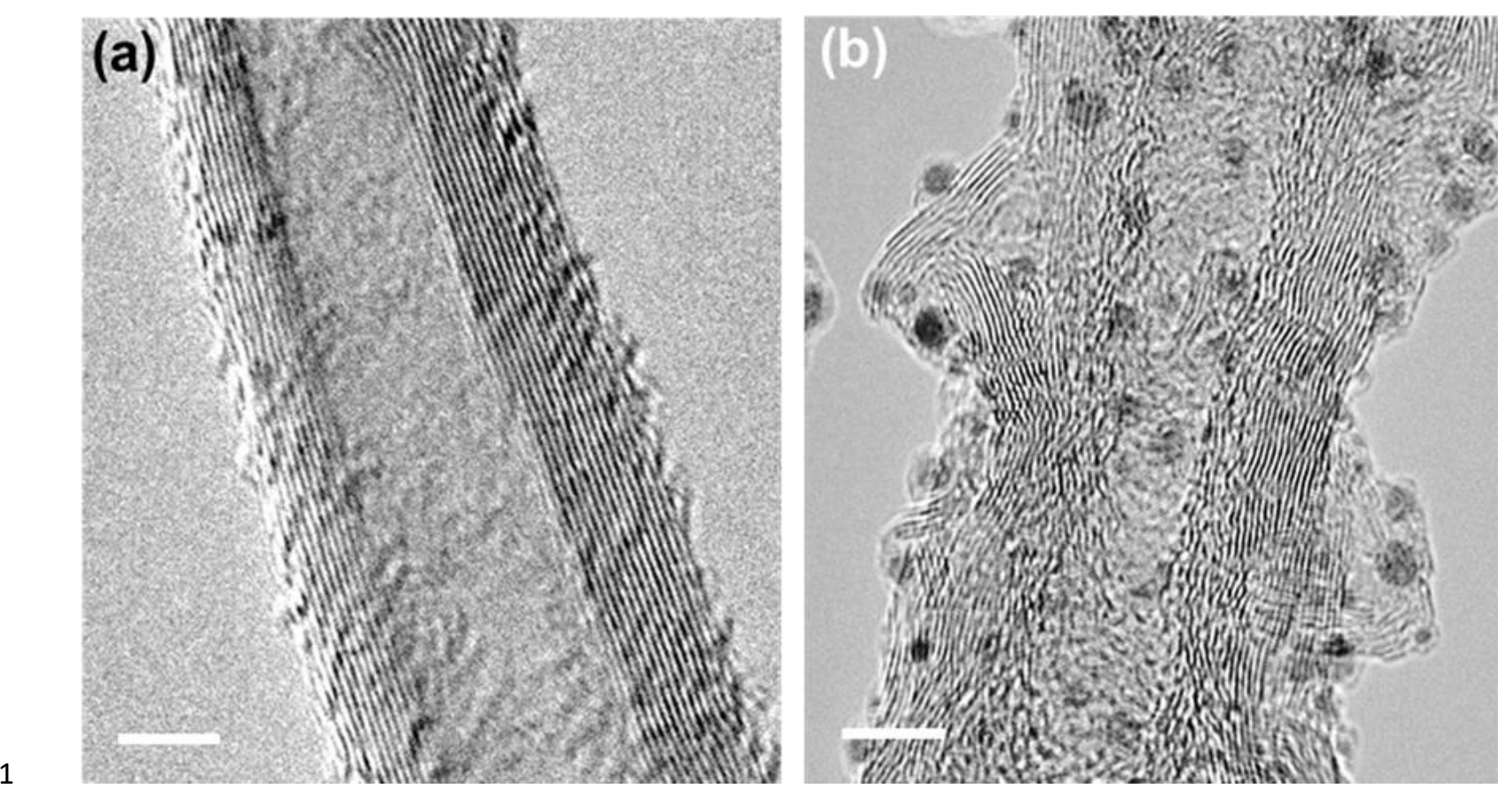

Figure 4. Nanoscale structural analysis of prepared VACNT-BN nanocomposites. HRTEM images of single (a) bare VACNT and (b) VACNT-BN nanocomposites. Scale bars: $5 \mathrm{~nm}$.

\subsection{Electrical Anisotropy}

Now that a kind of core-shell structure of the VACNT-BN nanocomposites prepared through our approach was confirmed, we explored its electrical potential performance towards interconnect applications. Two types of VACNT-BN test benches in similar sizes, with orthogonal orientations of VACNTs, were prepared. As can be seen in Figure 5a and 5b, the top view SEM images of the two test benches were (a) perpendicular to and (b) parallel to the VACNTs' direction, respectively. The insets present the overall view of these two test benches. The test benches were welded onto gold $(\mathrm{Au})$ electrodes in an all-around fashion to ensure good electrical connection. As can be seen in Figure 5a, the VACNTs were pointing downwards; and the current passed through the VACNTs horizontally. On the contrary, in Figure 5b, the VACNTs were pointing from left to right, parallel 
1 with the direction of the current. Interestingly, the current flowing through these two test benches

2 exhibited a huge difference, of two orders of magnitude, in electrical conductivity.

3 Figure $5 \mathrm{c}$ compares the I-V curves of VACNT-BN measured on both test benches, where the

4 blue and red lines represent the I-V parallel with the direction of VACNT and perpendicular to the

5 direction of the VACNT, respectively. The electrical conductivity turned out to be $1060.43 \mathrm{~S} \cdot \mathrm{m}^{-1}$

6 along the VACNT direction, while only $4.43 \mathrm{~S} \cdot \mathrm{m}^{-1}$ measured perpendicular to the VACNT

7 direction. Although the electrical conductivity of the VACNT-BN was significantly lower than bare

8 multiwalled carbon nanotube (MWCNT) bundles, the electrical conductivity of which was

9 reported to be $\sim 2.8 \cdot 10^{7} \mathrm{Sm}^{-1}$ at a length of $\sim 20 \mu \mathrm{m},{ }^{53}$ it showed superior electrical conductivity

10 over CNT-polymer mixtures, whose conductivities were usually below $10^{-3} \mathrm{~S} \cdot \mathrm{m}^{-1},{ }^{21,49}$ The sample

11 also demonstrated good thermal stability and robust electrical performance after being heat cycled

12 from $100{ }^{\circ} \mathrm{C}$ to $600{ }^{\circ} \mathrm{C}$ in argon flow, as shown in Figure $5 \mathrm{~d}$, which is significantly beneficial for

13 practical applications. In addition, the red line, representing the current perpendicular to the

14 direction of the VACNTs, showed some nonlinear characteristics. All of these results indicated the

15 existence of different conductive pathways inside the VACNT-BN system. 

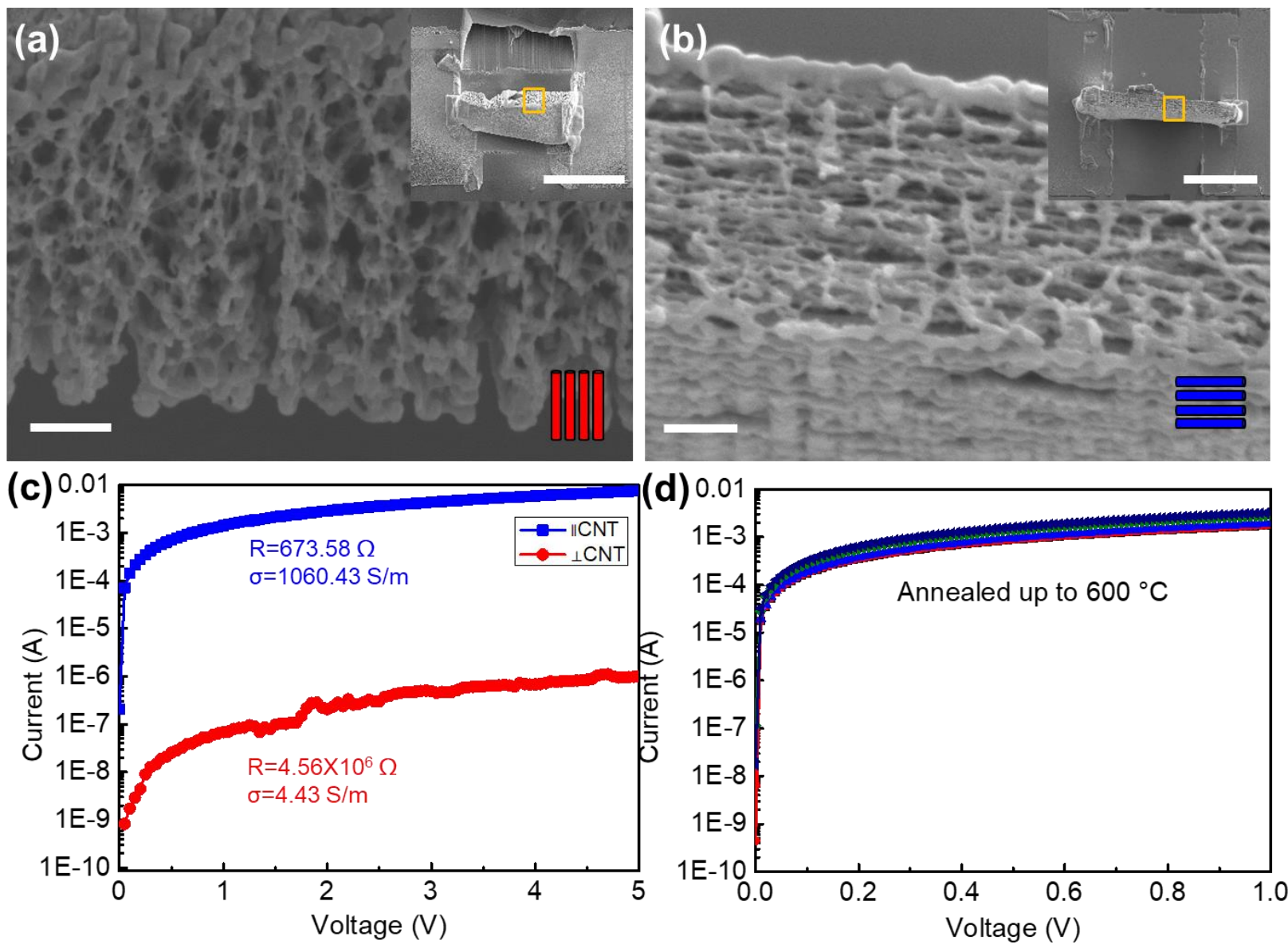

Figure 5. Electrical anisotropy of prepared VACNT-BN nanocomposites. $(a, b)$ VACNT-BN test benches for $\mathrm{I}-\mathrm{V}$ analysis where the direction of VACNTs is (a) perpendicular to and (b) parallel with the direction of the current flow. Insets present low magnification SEM images of the test benches. (c) I-V characteristics in log scale of (a) red and (b) blue. (d) I-V characteristics of (b) after annealing in argon flow from $100^{\circ} \mathrm{C}$ to $600^{\circ} \mathrm{C}$. Scale bars for (a) and (b): $500 \mathrm{~nm}$. Scale bars for insets of $(a, b): 15 \mathrm{um}$.

In an insulator-conductor system, multiple electron percolation thresholds could co-exist, due to the complex formation of the conducting network. ${ }^{55}$ To better understand this phenomenon, the percolation effect of electrons in our VACNT-BN nanocomposites was analyzed. In our VACNTBN system, the VACNTs were highly oriented and shielded by h-BN layers. Due to the dielectric nature of h-BN compared to the metallic VACNTs, the condition of electron transport was expected to be dominated by the pathways provided by the VACNTs themselves and mainly determined by 
1 the geometrical feature of individual VACNTs. Natsuki et al. investigated different electrical

2 percolation behaviors as a result of different orientations and aspect ratios of fillers. $\mathrm{Lu}$ et al.

3 compared various simulation works regarding conductive nanocomposites. ${ }^{51,52}$ In general, with an

4 increase in aspect ratio, an exponential decrease in the EPT was found, regardless of the waviness

5 of the CNTs. ${ }^{21,51}$ As the typical diameter of our sample CNTs was $\sim 20 \mathrm{~nm}$ (Figure $4 \mathrm{~b}$ ) and the

6 length of the CNTs in the test benches (Figure 5a and 5b) was $\sim 20 \mu \mathrm{m}$, the aspect ratio was

7 calculated to be 1000:1, which was significantly larger than the ones used in the simulation. ${ }^{21,51}$

8 Consequently, extremely conductive behavior along the direction of the VACNTs is expected.

9 As for the direction perpendicular to the VACNTs, the introduction of BN on the wall of the

10 VACNTs eliminated the contact between adjacent tubes. The extremely large band gap of BN made

11 it a perfect insulating layer as well. In this case, the intertube conduction of electrons was extremely

12 limited by the high barrier provided, which significantly increased the EPT, resulting in a

13 significantly lower electrical conductivity along this direction. Meanwhile, the electron conduction

14 along the VACNTs was not affected. Overall, VACNTs provided highly aligned electrical

15 conductive pathways while the infiltrated $\mathrm{h}-\mathrm{BN}$ in the nanocomposites induced the significant

16 difference in EPT along different directions, ${ }^{50}$ which comprehensively yielded the highly

17 anisotropic electrical conducting performance of the prepared VACNT-BN nanocomposites.

As a contrast, we also measured the conductivity of bare VACNTs from both the parallel and

19 perpendicular directions (Figure S11) and obtained very similar results although the geometrical

20 aspect ratios were also significantly different for the two directions. It suggests that other factors, 
besides the geometrical feature of individual VACNTs, take effect in determining the conductive behavior of bare VACNTs. In addition, the physical distortions of VACNTs can affect the electron transport in the $\mathrm{CNT},{ }^{53}$ which helps explain the lower electrical conductivity of VACNT-BN (along CNT direction) than bare VACNTs. It was reported that for randomly aligned CNTs, measuring direction does not affect conductivity by too much. ${ }^{20}$ For the bare VACNTs, multiple contact points actually existed among adjacent tubes. Thus, a complex and nondirectional conducting network formed, on which condition the conduction behavior of the electron was dominated not only by the pathways provided by individual VACNTs but also by the intertube percolation. As a result, no obvious anisotropy in the electrical conducting behaviors was measured for the bare VACNTs.

It can be noticed that the level of electrical conductivity of VACNT-BN comparing with CNTs and other conductive material is substantially lower. In spite of that, when comparing to CNTmixture counterparts, such as $\mathrm{CNT}-\mathrm{Al}_{2} \mathrm{O}_{3},{ }^{58,59} \mathrm{CNT}-\mathrm{MgO}^{59}$ and $\mathrm{CNT}-\mathrm{Si}_{3} \mathrm{~N}_{4},{ }^{60}$ we still demonstrate competitive electrical conductivity. In our present research, we mainly intend to demonstrate an approach which can effectively infiltrate BN to VACNT forest to improve its thermal stability and electrical anisotropy. Although it is still early to demonstrate an application-ready VACNT-BN system due to the limited absolute electrical conductivity value currently reached, there are possible ways to improve the electrical conductivity in future works, such as: (1) reducing the amount of $\mathrm{BN}$ in the system. By controlling the amount of the $\mathrm{BN}$ infiltrated into the VACNT forest, the insulating layers between tubes will be thinner and some degree of conduction will be promoted while maintaining good enough anisotropic conducting ability; (2) reducing the growth 
rate of $\mathrm{BN}$ for finer control of $\mathrm{BN}$ layers formed outside of the VACNT walls, to avoid

2 discontinuous $\mathrm{BN}$ formation and large defects. In addition, avoiding the formation of curvatures

3 of VACNTs could effectively reduce strain in VACNTs, which could increase electron mobility in

4 VACNTs $^{61}$ and (3) preparing better quality VACNTs. The quality improvement of bare VACNTs

5 can enhance its electrical conductivity, which should also be able to enhance the electrical

6 conductivity of the corresponding VACNT-BN nanocomposites.

\section{$7 \quad$ 3.4. Thermal Stability}

8 Previous studies showed moderate thermal stability and reasonable antioxidative ability of

9 VACNTs up until $600{ }^{\circ} \mathrm{C} .{ }^{28,62}$ Meanwhile, h-BN demonstrated very good antioxidative ability at

10 elevated temperatures. ${ }^{25}$ To verify this, the antioxidative stability of our as-grown h-BN film was

11 tested in air. Raman spectra were collected after each oxidation process. As shown in Figure S12a,

12 samples exhibited no evident change as the oxidation temperature increased to even $1100{ }^{\circ} \mathrm{C}$. We

13 further investigated the oxidation temperature dependence of peak position and full width at half

14 maximum (FWHM) of the $\mathrm{E}_{2 \mathrm{~g}}$ peak of h-BN. No obvious change was observed in either FWHM

15 or the peak position, indicating the high quality and high-temperature oxidation resistance of the

16 h-BN films grown by our approach.

17 The effect of oxidation time on the high-temperature oxidation resistance of the as-grown h-

$18 \mathrm{BN}$ films was also studied by treating them at $1100{ }^{\circ} \mathrm{C}$ in the air for an extended period (Figure

$19 \mathrm{~S} 13)$. The thickness of the h-BN films was evaluated after each oxidation process via cross-

20 sectional SEM. The original thickness of the h-BN film was $\sim 11.8 \mu \mathrm{m}$ (Figure S13a). After being 
oxidized at $1100{ }^{\circ} \mathrm{C}$ for $5 \mathrm{~min}$, the film thickness decreased slightly to $\sim 11.5 \mu \mathrm{m}$ (Figure $\mathrm{S} 13 \mathrm{~b}$ ). When the oxidization time was extended to $40 \mathrm{~min}$, the film thickness was decreased to $\sim 8 \mu \mathrm{m}$, suggesting a slow oxidation rate at $1100{ }^{\circ} \mathrm{C}$. In spite of this, the oxidation only happened on the upper section of the layered h-BN, indicating it was a good candidate for the protective layer for the VACNTs.

Although the antioxidative ability of $\mathrm{h}-\mathrm{BN}$ has been determined, the effect of infiltrating $\mathrm{h}-\mathrm{BN}$ into VACNT arrays is still unknown. Therefore, we further investigated the antioxidative ability of as-prepared VACNT-BN nanocomposites. The VACNT-BN nanocomposites were first oxidized at $1100{ }^{\circ} \mathrm{C}$ for a long treatment time of $40 \mathrm{~min}$ in air. Figure $\mathrm{S} 14 \mathrm{a}$ and $\mathrm{S} 14 \mathrm{~b}$ compare the change in sample thickness (a) before and (b) after the high-temperature oxidation treatment. The thickness of the sample decreased by $\sim 7 \mu \mathrm{m}$ in total after $40 \mathrm{~min}$ of oxidation at $1100{ }^{\circ} \mathrm{C}$. Encouragingly, the oxidation process demonstrated behavior similar to the h-BN layer, which showed that the decrease in thickness only happened in the upper section of the thick h-BN film. Meanwhile, the lower VACNT-BN area was not affected at all. An enlarged SEM image in Figure S14c shows the layered h-BN film near the top surface to be covered with multiple visible rough oxidation sites, suggesting that the oxidation started from the top surface of the h-BN. Scanning electron microscopy images in Figure S14d-S14f show no visual evidence of oxidation in the original VACNT area, indicating a good protection effect provided by the infiltrated h-BN. The thickness decrease under the high-temperature, oxidation condition in the upper h-BN film was expected and could be improved by increasing the thickness of the upper h-BN further, if needed. It's worthy of noting that, during the measurement of electrical conductivity of our VACNT-BN 
1 nanocomposites, we need to expose the CNT tips to ensure good electrical contact. However,

2 during practical applications, BN will be deposited to cover not only the VACNTs, but also the

3 electrical contact and corresponding mating parts, so that its refractory ability and protective effect

$4 \quad$ can be sufficiently utilized.
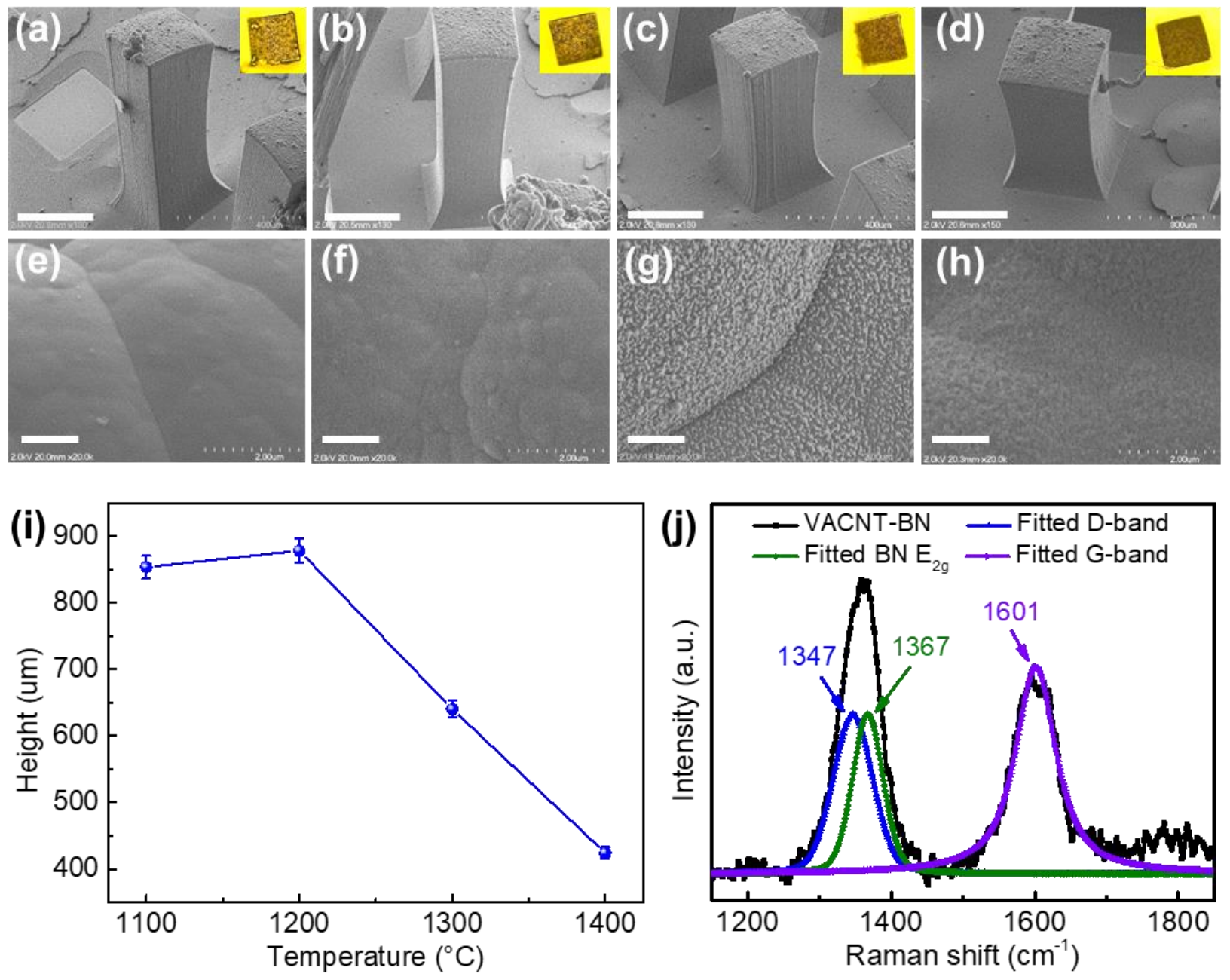

Figure 6. Antioxidative ability of prepared VACNT-BN nanocomposites. (a-d) SEM images of VACNT-BN

cuboid samples after oxidation at (a) $1100^{\circ} \mathrm{C}$, (b) $1200^{\circ} \mathrm{C}$

${ }^{\circ} \mathrm{C}$, (c) $1300{ }^{\circ} \mathrm{C}$ and (d) $1400^{\circ} \mathrm{C}$. (e-h) Magnified SEM images of the top area of the samples from (a) to (d), respectively. (i) Height change of VACNT-BN cuboid after oxidation. (j) Raman spectrum of VACNT-BN after being oxidized for 30 min at $1400^{\circ} \mathrm{C}$. Fitted lines represent individual peaks of interests.

Since h-BN can be stable up to much higher temperatures, the antioxidative ability of the 
1 VACNT-BN nanocomposites was examined under even harsher conditions, including ultrahightemperature oxidation treatment (up to $1400^{\circ} \mathrm{C}$ ) and an Ultrahigh-temperature gas erosion test. To better visually observe the oxidation results, VACNT-BN samples in the cubic pattern, instead of

4 films, were used and oxidized from $1100^{\circ} \mathrm{C}$ to $1400^{\circ} \mathrm{C}$, for $5 \mathrm{~min}$ at each temperature, with oxygen 5 partial pressure of 100 mTorr (Figure 6a-6d, respectively). Magnified SEM images of the top 6 surface are shown in Figure 6e-6h, corresponding to Figure 6a-6d. As can be seen, the originally 7 smooth surface became rough as the oxidation temperature increased, and significant surface morphology change was observed beginning at $1300{ }^{\circ} \mathrm{C}$ (Figure $6 \mathrm{~g}$ ). Meanwhile, the height of 9 each VACNT-BN cuboids started to drastically reduce after $1300^{\circ} \mathrm{C}$ (Figure 6i), indicating the loss of VACNTs during the oxidation. It has been reported that $\mathrm{h}-\mathrm{BN}$ are prone to oxidize under this

11 condition accompanied by a slight weight gain and a decrease in size. ${ }^{63}$ The shrinkage of the 12 outside BN caused thinning of the protective BN layer, eventually exposing the inside VACNTs to 13 the environment. In addition, Raman characterization of samples in figures 6a-6d are shown in 14 figure S15. Comparing to the Raman spectrum of the sample before any oxidation treatment (figure $15 \mathrm{~S} 15 \mathrm{a})$, the combined feature $\mathrm{D}+\mathrm{E}_{2 \mathrm{~g}}$ around $1370 \mathrm{~cm}^{-1}$ demonstrate no significant loss in intensity and a stable peak position. The G-band associating to CNTs, which is around $1580 \mathrm{~cm}^{-1}$, gained

17 slightly change in intensity and became more pronounced after oxidation. The peak position of Gband maintained relatively stable after 5 min of oxidation at each temperature. When prolonging 19 the oxidation duration to $30 \mathrm{~min}$ at $1400{ }^{\circ} \mathrm{C}$, the G-band blue-shifted to $1601 \mathrm{~cm}^{-1}$. One of the 20 possible reasons for such a blueshift of the G-band is that the thermal expansion coefficient of $21 \mathrm{CNT}$ (positive) and BN (negative) are mismatched. When heating for a longer time, the BN layers 
compress the inside CNTs, causing the increase in the Raman shift. Despite this, the overall chemical composition stayed unchanged, which indicates that the outside BN layers can protect the inside VACNTs well at temperature as high as $1400^{\circ} \mathrm{C}$. The result of oxidation of VACNT-BN nanocomposites is a material loss rather than chemical change since carbon and boron does not react to form any side products like B-C-N. Thus, the oxidation cannot be reflected in the Raman spectroscopy. It is this material loss caused by oxidation as well as the shrinkage of BN under oxidative environment who should account for the significant decrease of the height of VACNTBN nanocomposites after $1300{ }^{\circ} \mathrm{C}$.

An ultrahigh-temperature gas erosion test was performed as well. Figure S16a and S16b show the actual experimental setup and an illustration of the method, respectively. A high-velocity gas flame $(2480 \mathrm{sccm})$ with an ultrahigh temperature (up to $3000{ }^{\circ} \mathrm{C}$ ) was kept flowing toward the surface of the VACNT-BN sample for $126 \mathrm{~min}$ in total. The average height change of the VACNTBN cuboids on the substrate was estimated by a Zygo NewView ${ }^{\mathrm{TM}}$ optical surface profiler, and the results are shown in Figure S16c. The average height did not change significantly after 126 min of erosion, and the optical images did not show obvious morphology changes either, as shown in the Figure S16c inset. Raman spectra at slightly different stages of the erosion test are shown in Figure S16d. As can be seen, the main features, namely, the D- and G-band for VACNT, as well as the $\mathrm{E}_{2 \mathrm{~g}}$ band for h-BN, presented no apparent changes, confirming good erosion resistance of the prepared VACNT-BN nanocomposites against oxygen contained in the ultrahigh temperature, high-velocity gas flow. As a comparison, no on par stability performance for the CNT-polymer-based 
1 nanocomposites has been reported as yet. The excellent stability of VACNT-BN as electrical

2 interconnects demonstrates great potential for high-power and high-current density applications,

3 such as insulated-gate bipolar transistors (IGBT).

\section{4. CONCLUSIONS}

5 In summary, a novel approach has been demonstrated for the finely controlled fabrication of 6 refractory VACNT-BN conductive nanocomposites. Ultralong VACNTs (up to $3 \mathrm{~mm}$ ), as

7 superaligned conducting pathways and building framework templates, were prepared based on

8 which uniformly infiltrated VACNT-BN composite structures were successfully obtained via the

9 one-step CVD process. The as-fabricated VACNT-BN nanocomposites exhibited significant

10 anisotropic electrical conducting performance, excellent thermal stability, and the extraordinary

11 ability to withstand various extreme conditions (e.g., ultrahigh-temperature oxidation, and high-

12 velocity hot gas erosion). The h-BN layers which were uniformly infiltrated into the VACNT arrays

13 significantly reduced the intertube electron percolation, providing excellent stability enhancement

14 at the same time. These attributes of VACNT-BN nanocomposites demonstrated great potential to

15 be superior to many CNT-based counterparts for high density interconnect applications. In addition,

16 the CVD process proposed in this work requires minimal complexity and effort in sample

17 preparation, which is promising for the development of a cost-effective, turnkey solution to

18 fabrication of scalable electrical interconnects. Our future work will explore the potential 19 applications for VACNT-BN nanocomposites and their properties under extreme conditions. 


\section{ASSOCIATED CONTENT}

2 Supporting Information. The Supporting Information is available free of charge on the ACS

3 Publications website at http://pubs.acs.org.

4 (Figure S1) Characterization of grown h-BN films; (Figure S2) Cross-sectional Raman mapping 5 of grown h-BN film; (Figure S3) TEM and EELS characterization of BN grown on SiO2/Si. ;

6 (Figure S4) XRD spectra of VACNTs, h-BN, and VACNT-BN; (Figure S5) Morphology

7 characterization of VACNT-BN composite; (Figure S6) Cross-sectional SEM images of single

8 ultralong VACNT-BN cuboid structure, top to bottom; (Figure S7) Cross-sectional SEM images

9 of a single ultralong VACNT-BN cuboid structure, side to side; (Figure S8) Cross-sectional Raman mapping characterization of short VACNT-BN composite; (Figure S9) Scanning TEM EELS mapping of VACNT-BN; (Figure S10) HRTEM of VACNT-BN and h-BN; (Figure S11) Electrical conductivity of VACNTs along two different directions; (Figure S12) Raman characterization of as-grown $\mathrm{BN}$ films after high-temperature oxidation treatments; (Figure S13) Cross-sectional SEM images of the thick BN film; (Figure S14) High-temperature antioxidation ability of VACNTBN nanocomposites; (Figure S15) Raman characterization of VACNT-BN after high-temperature oxidation treatments; (Figure S16) Ultrahigh-temperature gas erosion test of CNT-BN.

\section{AUTHOR INFORMATION}

18 * Corresponding Author

19 E-mail: ylu2@unl.edu

20 E-mail: fanpeixun@gmail.com 


\section{CONFLICTS OF INTEREST}

2 The authors declare no conflict of interest.

3 NOTES

$4{ }^{ \pm}$Authors contributed equally.

5 ACKNOWLEDGMENTS

6 This research was financially supported by U.S. Department of Energy [NETL: DE-FE0023061],

7 and the Nebraska Center for Energy Science Research. Manufacturing and characterization

8 analyses were performed at the NanoEngineering Research Core Facility (part of the Nebraska

9 Nanoscale Facility), which is partially funded by the Nebraska Research Initiative. 


\section{References}

(1) http://www-03.ibm.com/ibm/history/ibm100/us/en/icons/copperchip/

(2) Bohr, M. T. Interconnect Scaling-the Real Limiter to High Performance ULSI. Proc. Int. Electron Devices Meet. 1995, 95, 241-244.

(3) Yee, C. F.; Jambek, A. B.; Al-Hadi, A. A. Advantages and Challenges of 10-Gbps Transmission on High-Density Interconnect Boards. J. Electron. Mater. 2016, 45, 31343141.

(4) Holden, H.; Andresakis, J.; Bogatin, E.; Carano, M.; Carpenter, K. A.; Dietz, K. H.; Laing, M.; Vaucher, C.; Viklund, P.; Wuensch, M. The HDI Handbook, 1st ed.; El Dorado Hills: BR, 2009.

(5) https://www.ncabgroup.com/hdi-pcb/

(6) Romero, C.; Park, S.; Kweon, Y.; Park, M. Advanced High Density Interconnection Substrate for Mobile Platform Application. Proc. Tech. Pap. - Int. Microsystems, Packag. Assem. Circuits Technol. Conf. IMPACT 2011, 214-217.

(7) Andricacos, P. C.; Uzoh, C.; Dukovic, J. O.; Horkans, J.; Deligianni, H. Damascene Copper Electroplating for Chip Interconnections. IBM J. Res. Dev. 1998, 42, 567-574.

(8) Chai, Y.; Chan, P. C. H. H. High Electromigration-Resistant Copper/Carbon Nanotube Composite for Interconnect Application. IEEE IEDM Technical Digest 2008.

(9) Wei, B. Q.; Vajtai, R.; Ajayan, P. M. Reliability and Current Carrying Capacity of Carbon Nanotubes. Appl. Phys. Lett. 2001, 79, 1172-1174.

(10) Kim, Y. L.; Li, B.; An, X.; Hahm, M. G.; Chen, L.; Washington, M.; Ajayan, P. M.; Nayak, 
S. K.; Busnaina, A.; Kar, S.; Jung, Y. J. Highly Aligned Scalable Platinum- Decorated Single-Wall Carbon Nanotube Interconnects. ACS Nano 2009, 3, 2818-2826.

(11) Kreupl, F.; Graham, A. P. A. P.; Duesberg, G. S. G.; Steinhögl, W.; Liebau, M.; Unger, E.; Hönlein, W. Carbon Nanotubes in Interconnect Applications. Microelectron. Eng. 2002, 64, 399-408.

(12) Nieuwoudt, A.; Massoud, Y. Evaluating the Impact of Resistance in Carbon Nanotube Bundles for VLSI Interconnect Using Diameter-Dependent Modeling Techniques. IEEE Trans. Electron Devices 2006, 53, 2460-2466.

(13) Li, J.; Ye, Q.; Cassell, A.; Ng, H. T.; Stevens, R.; Han, J.; Meyyappan, M. Bottom-up Approach for Carbon Nanotube Interconnects. Appl. Phys. Lett. 2003, 82, 2491-2493.

(14) Homma, Y.; Yamashita, T.; Kobayashi, Y.; Ogino, T. Interconnection of Nanostructures Using Carbon Nanotubes. Phys. B Condens. Matter 2002, 323, 122-123.

(15) Naeemi, A.; Meindl, J. D. Monolayer Metallic Nanotube Interconnects: Promising Candidates for Short Local Interconnects. IEEE Electron Device Lett. 2005, 26, 544-546.

(16) Sahoo, S.; Chitturi, V. R.; Agarwal, R.; Jiang, J. Thermal Conductivity of Freestanding Single Wall Carbon Nanotube Sheet by Raman Spectroscopy. ACS Appl. Mater. Interfaces 2014, 6, 19958-19965.

(17) Hao, M.; Kumar, A.; Hodson, S. L.; Zemlyanov, D.; He, P.; Fisher, T. S. Brazed Carbon Nanotube Arrays: Decoupling Thermal Conductance and Mechanical Rigidity. Adv. Mater. Interfaces 2017, 4, 1601042.

(18) Behnam, A.; Guo, J.; Ural, A. Effects of Nanotube Alignment and Measurement Direction 
on Percolation Resistivity in Single-Walled Carbon Nanotube Films. J. Appl. Phys. 2007, 102, 044313.

(19) Wu, S. H.; Masaharu, I.; Natsuki, T.; Ni, Q. Q. Electrical Conduction and Percolation Behavior of Carbon Nanotubes/UPR Nanocomposites. J. Reinf. Plast. Compos. 2006, 25, 1957-1966.

(20) Simoneau, L. P.; Villeneuve, J.; Rochefort, A. Electron Percolation in Realistic Models of Carbon Nanotube Networks. J. Appl. Phys. 2015, 118, 124309.

(21) Zeng, X.; Xu, X.; Shenai, P. M.; Kovalev, E.; Baudot, C.; Mathews, N. Characteristics of the Electrical Percolation in Carbon Nanotubes / Polymer Nanocomposites. J. Phys. Chem. C 2011, 115, 21685-21690.

(22) Vigolo, B. An Experimental Approach to the Percolation of Sticky Nanotubes. Science. 2005, 309, 920-924.

(23) Bu, H.; Zhao, M.; Zhang, H.; Wang, X.; Xi, Y.; Wang, Z. Isoelectronic Doping of Graphdiyne with Boron and Nitrogen: Stable Configurations and Band Gap Modification. J. Phys. Chem. A 2012, 116, 3934-3939.

(24) Zhang, J.; Yang, Y.; Lou, J. Investigation of Hexagonal Boron Nitride as an Atomically Thin Corrosion Passivation Coating in Aqueous Solution. Nanotechnology 2016, 27, 364004.

(25) Kostoglou, N.; Polychronopoulou, K.; Rebholz, C. Thermal and Chemical Stability of Hexagonal Boron Nitride (h-BN) Nanoplatelets. Vacuum 2015, 112, 42-45.

(26) Cassabois, G.; Valvin, P.; Gil, B. Hexagonal Boron Nitride Is an Indirect Bandgap 
Semiconductor. Nat. Photonics 2016, 10, 262-266.

(27) Li, W. Z.; Wen, J. G.; Sennett, M.; Ren, Z. F. Clean Double-Walled Carbon Nanotubes Synthesized by CVD. Chem. Phys. Lett. 2003, 368, 299-306.

(28) Zou, Q. M.; Deng, L. M.; Li, D. W.; Zhou, Y. S.; Golgir, H. R.; Keramatnejad, K.; Fan, L. S.; Jiang, L.; Silvain, J. F.; Lu, Y. F. Thermally Stable and Electrically Conductive, Vertically Aligned Carbon Nanotube/Silicon Infiltrated Composite Structures for HighTemperature Electrodes. ACS Appl. Mater. Interfaces 2017, 9, 37340-37349.

(29) Jing, L.; Tay, R. Y.; Li, H.; Tsang, S. H.; Huang, J.; Tan, D.; Zhang, B.; Teo, E. H. T.; TOK, A. I. Y. Coaxial Carbon@boron Nitride Nanotube Arrays with Enhanced Thermal Stability and Compressive Mechanical Properties. Nanoscale 2016, 8, 11114-11122.

(30) Jing, L.; Samani, M. K.; Liu, B.; Li, H.; Tay, R. Y.; Tsang, S. H.; Cometto, O.; Nylander, A.; Liu, J.; Teo, E. H. T.; Tok, A. I. Y. Thermal Conductivity Enhancement of Coaxial Carbon@Boron Nitride Nanotube Arrays. ACS Appl. Mater. Interfaces 2017, 9, 1455514560.

(31) Stano, K. L.; Carroll, M.; Padbury, R.; McCord, M.; Jur, J. S.; Bradford, P. D. Conformal Atomic Layer Deposition of Alumina on Millimeter Tall, Vertically-Aligned Carbon Nanotube Arrays. ACS Appl. Mater. Interfaces 2014, 6, 19135-19143.

(32) Pierson, H. O. Boron Nitride Composites By Chemical Vapor Deposition. J. Compos. Mater. 1975, 9, 228-240.

(33) Li, L. H.; Chen, Y. Atomically Thin Boron Nitride: Unique Properties and Applications. Adv. Funct. Mater. 2016, 26, 2594-2608. 
(34) Zhi, C.; Bando, Y.; Tang, C.; Kuwahara, H.; Golberg, D. Large-Scale Fabrication of Boron Nitride Nanosheets and Their Utilization in Polymeric Composites with Improved Thermal and Mechanical Properties. Adv. Mater. 2009, 21, 2889-2893.

(35) Xue, Y.; Liu, Q.; He, G.; Xu, K.; Jiang, L.; Hu, X.; Hu, J. Excellent Electrical Conductivity of the Exfoliated and Fluorinated Hexagonal Boron Nitride Nanosheets. Nanoscale Res. Lett. 2013, 8, 1-7.

(36) Arenal, R.; Lopez-Bezanilla, A. In-Situ Formation of Carbon Nanotubes Encapsulated within Boron Nitride Nanotubes via Electron Irradiation. ACS Nano 2014, 8, 8419-8425.

(37) Zhang, G.; Zhou, R.; Zeng, X. C. Carbon Nanotube and Boron Nitride Nanotube Hosted C60-V Nanopeapods. J. Mater. Chem. C 2013, 1, 4518-4526.

(38) An, W.; Turner, C. H. Linking Carbon and Boron-Nitride Nanotubes: Heterojunction Energetics and Band Gap Tuning. J. Phys. Chem. Lett. 2010, 1, 2269-2273.

(39) Walker, K. E.; Rance, G. A.; Pekker, Á.; Tóháti, H. M.; Fay, M. W.; Lodge, R. W.; Stoppiello, C. T.; Kamarás, K.; Khlobystov, A. N. Growth of Carbon Nanotubes inside Boron Nitride Nanotubes by Coalescence of Fullerenes: Toward the World's Smallest Coaxial Cable. Small Methods 2017, 1, 1700184.

(40) Miranda, A. M.; Castilho-Almeida, E. W.; Martins Ferreira, E. H.; Moreira, G. F.; Achete, C. A.; Armond, R. A. S. Z. S. Z.; Dos Santos, H. F.; Jorio, A.; Martins, E. H.; Moreira, G. F.; Achete, C. A.; Armond, R. A. S. Z. S. Z.; Dos, H. F.; Jorio, A. Line Shape Analysis of the Raman Spectra from Pure and Mixed Biofuels Esters Compounds. Fuel 2014, 115, 118125. 
1 (41) Jesson, D. E.; Pennycook, S. J. Incoherent Im Aging of Crystals Using Therm Ally Scattered

Electrons. Proc. R. Soc. Lond. A 1995, 449, 273-293.

(42) Mehrtens, T.; Schowalter, M.; Tytko, D.; Choi, P.; Raabe, D.; Hoffmann, L.; Jönen, H.;

Rossow, U.; Hangleiter, A.; Rosenauer, A. Measuring Composition in InGaN from

HAADF-STEM Images and Studying the Temperature Dependence of Z-Contrast. In Journal of Physics: Conference Series 2013, 471, 012009.

(43) Wang, J.; Hao, J.; Liu, D.; Qin, S.; Portehault, D.; Li, Y.; Chen, Y.; Lei, W. Porous Boron Carbon Nitride Nanosheets as Efficient Metal-Free Catalysts for the Oxygen Reduction Reaction in Both Alkaline and Acidic Solutions. ACS Energy Lett. 2017, 2, 306-312.

(44) Lei, W.; Portehault, D.; Dimova, R.; Antonietti, M. Boron Carbon Nitride Nanostructures from Salt Melts: Tunable Water-Soluble Phosphors. J. Am. Chem. Soc. 2011, 133, 71217127.

(45) Kosaka, M.; Urakami, N.; Hashimoto, Y. Formation of Graphitic Carbon Nitride and Boron Carbon Nitride Film on Sapphire Substrate. In Japanese Journal of Applied Physics 2018, 57, $02 \mathrm{CB} 09$.

(46) Yap, Y. K. B-C-N Nanotubes and Related Nanostructures; Springer-Verlag, Berlin, Germany, 2009.

(47) Dobigeon, N.; Brun, N. Ultramicroscopy Spectral Mixture Analysis of EELS SpectrumImages. Ultramicroscopy 2012, 120, 25-34.

(48) Cretu, O.; Lin, Y. C.; Koshino, M.; Tizei, L. H. G.; Liu, Z.; Suenaga, K. Structure and Local Chemical Properties of Boron-Terminated Tetravacancies in Hexagonal Boron Nitride. 
Phys. Rev. Lett. 2015, 114, 075502.

(49) Nag, A.; Raidongia, K.; Hembram, K. P. S. S.; Datta, R.; Waghmare, U. V.; Rao, C. N. R. Graphene Analogues of BN: Novel Synthesis and Properties. ACS Nano 2010, 4, 15391544.

(50) Yun, J.; Oh, H.; Jo, J.; Hwi Lee, H.; Kim, M.; Yi, G.-C. Selective-Area Heteroepitaxial Growth of h -BN Micropatterns on Graphene Layers. 2D Mater. 2017, 5, 015021.

(51) Yasuda, A.; Kawase, N.; Banhart, F.; Mizutani, W.; Shimizu, T.; Tokumoto, H. Graphitization Mechanism during the Carbon-Nanotube Formation Based on the in-Situ HRTEM Observation. J. Phys. Chem. B 2002, 106, 1849-1852.

(52) Chang, H.; Tsai, H.; Lin, W.; Chu, Y.; Hsu, W. Hexagonal Boron Nitride Coated Carbon Nanotubes: Interlayer Polarization Improved Field Emission. ACS Appl. Mater. Interfaces 2015, 7, 14456-14462.

(53) Lekawa-Raus, A.; Patmore, J.; Kurzepa, L.; Bulmer, J.; Koziol, K. Electrical Properties of Carbon Nanotube Based Fibers and Their Future Use in Electrical Wiring. Adv. Funct. Mater. 2014, 24, 3661-3682.

(54) McClory, C.; McNally, T.; Baxendale, M.; Potschke, P.; Blau, W.; Ruether, M. Electrical and Rheological Percolation of PMMA/MWCNT Nanocomposites as a Function of CNT Geometry and Functionality. Eur. Polym. J. 2010, 46, 854-868.

(55) Kovacs, J. Z.; Velagala, B. S.; Schulte, K.; Bauhofer, W. Two Percolation Thresholds in Carbon Nanotube Epoxy Composites. Compos. Sci. Technol. 2007, 67, 922-928.

(56) Lu, W.; Chou, T. W.; Thostenson, E. T. A Three-Dimensional Model of Electrical 
Percolation Thresholds in Carbon Nanotube-Based Composites. Appl. Phys. Lett. 2010, 96, 223106.

(57) Natsuki, T.; Endo, M.; Takahashi, T. Percolation Study of Orientated Short-Fiber Composites by a Continuum Model. Phys. A Stat. Mech. its Appl. 2005, 352, 498-508.

(58) Inam, F.; Yan, H.; Jayaseelan, D. D.; Peijs, T.; Reece, M. J. Electrically Conductive Alumina-Carbon Nanocomposites Prepared by Spark Plasma Sintering. J. Eur. Ceram. Soc. 2010, 30, 153-157.

(59) Flahaut, E.; Peigney, A.; Laurent, C.; Marlière, C.; Chastel, F.; Rousset, A. Carbon Nanotube-Metal-Oxide Nanocomposites: Microstructure, Electrical Conductivity and Mechanical Properties. Acta Mater. 2000, 48, 3803-3812.

(60) Balázsi, C.; Kónya, Z.; Wéber, F.; Biró, L. P.; Arató, P. Preparation and Characterization of Carbon Nanotube Reinforced Silicon Nitride Composites. Mater. Sci. Eng. C 2003, 23, $1133-1137$.

(61) Hirai, H.; Ogawa, M.; Souma, S. Effect of Strain on Electron Mobility in Graphene. IEEE SISPAD 2017, 209-212.

(62) Frank, B.; Rinaldi, A.; Blume, R.; Schlögl, R.; Su, D. S. Oxidation Stability of Multiwalled Carbon Nanotubes for Catalytic Applications. Chem. Mater. 2010, 22, 4462-4470.

(63) Jacobson, N.; Farmer, S.; Lavrenko, V. A.; Alexeev, A. F. High-Temperature Oxidation of Boron Nitride. Ceram. Int. 1986, 12, 25-31. 
3 Qi Ming Zou, Lei Min Deng, Pei Xun Fan, * Da Wei Li, Chen Fei Zhang, Li Sha Fan, Lan Jiang,

4 Jean-Francois Silvain and Yong Feng Lu*

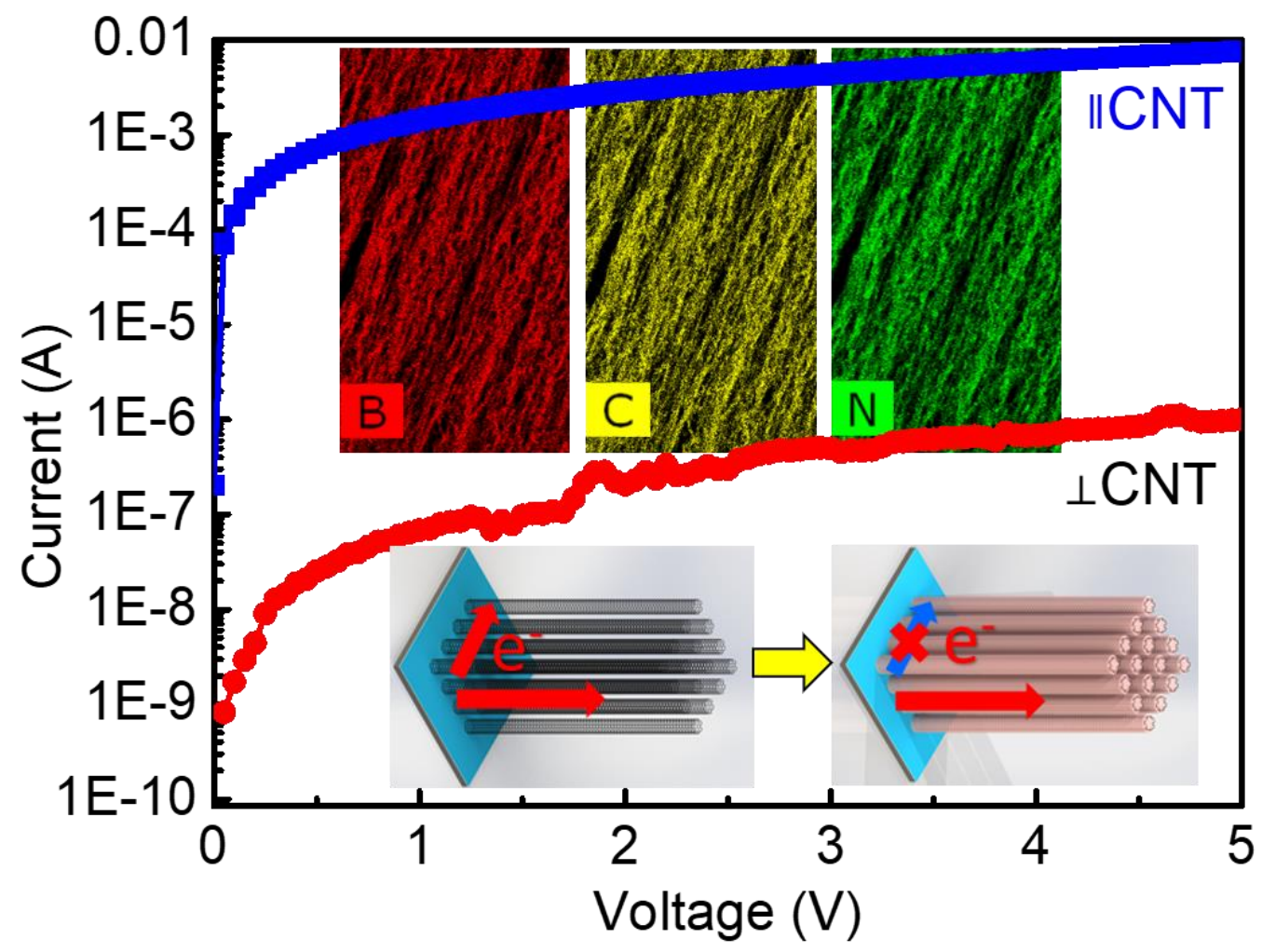

7

8 Highly anisotropic electrical conducting VACNT-BN nanocomposites were fabricated via fine 9 controlled infiltration of BN into the VACNT arrays. The anisotropic conducting behavior was a 10 result of the manipulated electron percolation thresholds in different directions, which essentially 11 allowed current flow along the direction of VACNTs. 